\title{
DETERMINATION OF THE DIPOLE MOMENT OF OCS WITH A MICLOWAVE ABSORPTION CAVITY
}

\author{
H. A. DIJKERMAN and G. RUITENBERG \\ Fysisch Laboralorium. Rijks-Universiteit, Utrecht, The Netherlands
}

Received 10 February 1959

\begin{abstract}
The electric dipole moment of OCS has been measured with a resonance cavity as a Stark absorption cell. The result: $\mu_{O C S}=0.7149 \pm 0.0003$. Debye agrees well with the dipole momant data cbtained with an electric resonance beam experiment.
\end{abstract}

\section{INTRODUCTION}

Recently Muenter [1] has measured the electric dipole moment of OCS using an electric resonance beam spectrometer. The result: $\mu_{\text {ocs }}=$ $=0.71521 \pm 0.00020$ Debye from the transition $(J, M)=(1,0) \rightarrow(1,1)$ is about $0.4 \%$ higher than that reported by Marshall et al. [2]. As the dipole moment of OCS is frequently used as a standard in microwave spectroscopy [3] it seemed to be worthwhile to measure it again with an independent method and different apparatus. So we used a resonance cavity as an absorption cell. The result of our measurements was: $\mu_{\text {OCS }}=0.7149 \pm 0.0003$ Debye. A description of the exper imental method and a discussion of the resulss is given.

\subsection{The cavily absorption cell}

Some years ago one of us has done measurements on intensity and saturation of microwaye absorption lines using a resonant cavity as a Stark absorption cell $[4,5]$. It was pointed out then that such a cavity was very suitable for absolute measurements on dipole moments [5] with a precision of better than $1: 10^{3}$. Therefore, and in order to check whether there was a discrepancy with the results of Muenter or not, we resumed these measurements. A description of the construction and performance of the cavity has already been reported elsewhere [6]. The essential features of this cavity designed for precision measurements on the Stark effect of microwave absorption lines, are:

a) An accurate determination of the height of the cavity (and thus of the distance between the Stark electrodes) is possible since resonances corresponding to several $\mathrm{TE}_{\mathrm{O}} \mathrm{mn}^{- \text {modes can be }}$ observed $(1 \leqslant m \leqslant 10 ; n=1,2, \ldots)$ by adjustment of the plunger of the cavity and reading of its position on a precision dial. In this way the height can be determined within about $2 \mu \mathrm{m}$, using the fact that the height for a typical $\mathrm{TE}_{0 m} 2^{- \text {reso- }}$ nance is twice the height for the corresponding

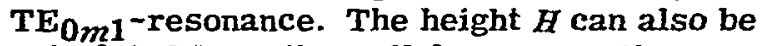
calculated from the well-known equation:

$$
\left(\frac{\nu}{c}\right)^{2}=\left(\frac{x_{0} m}{\pi D}\right)^{2}+\left(\frac{n}{2 H}\right)^{2}
$$

with: $\nu=$ resonance frequency of the cavity; $c^{2}=$ velocity of light; $x_{0 m}=m$-th zero of the Bessel function $J_{o}(x) ; D$ and $H$ are the diameter and height of the cavity, respectively.

If the frequency and the diameter are known, the height can thus be calculated. In table 1 the measured and calculated $H$ at the resonance frequency of the transition $J=1-2$ of OCS are shown. It can be concluded that the absolute inaccuracy of $H$ amounts to $2 \mu \mathrm{m}$; i.e. a precision of about $1: 5 \times 10^{3}$.

b) The homogeneity of the Stark field ( $D=150 \mathrm{~mm}, H \approx 10 \mathrm{~mm}$ ) prevents a strong broadening of the Stark component. A careful examination of the cavity bottom showed an unevenness of about $20 \mu \mathrm{m}$, irregularly distributed over the surface; however, the influence on the frequency shift of the Stark component is averaged out by the exciting microwave field which has a cylindrical symmetry around the axis of the cavity. The only effect of this inhomogeneity is a broadening of the Stark components. Moreover, this effect can relatively be reduced by increasing the height of the cavity. 
Table 1

Calculated and measured heights of the cavity $(D=150 \mathrm{~mm})$ at several $\mathrm{TE}_{0 \mathrm{mn}}$-modes.

\begin{tabular}{ccc}
\hline Mode: $\mathrm{TE}_{0 \mathrm{mn}}$ & $H_{\text {meas }}(\mathrm{mm})$ & $H_{\mathrm{cala}}(\mathrm{mm})$ \\
\hline 021 & 6.271 & 6.268 \\
031 & 6.394 & 6.392 \\
041 & 6.573 & 6.574 \\
051 & 6.826 & 6.826 \\
061 & 7.175 & 7.176 \\
071 & 7.663 & 7.665 \\
081 & 8.369 & 8.372 \\
\hline
\end{tabular}

\section{EXPERIMENTAL}

The frequency of the reflex klystron (OKI $24 \mathrm{~V} 10 \mathrm{~A}$ ) used in the experiment was stabilized with the aid of a frequency generator (Rhode and Schwartz, XUC) and a discriminator (Schomandl, FDS-30). With these units the frequency of the reflex klystron could be stabilized and measured with a precision of about $1 \mathrm{kHz}$.

In this way the klystron-frequency was preset at a fixed value. Next the absorption cavity was tuned to resonance at this frequency in a partic-

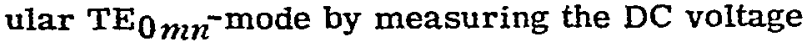
at the $X$-tal detector connected to the output waveguide of the cavity. The height of the cavity was read on the dial at the plunger. After that the modulated Stark voltage, consisting of a small square wave $10 \mathrm{kHz}$ voltage superimposed on a high adjustable DC voltage, was applied to the plunger. The output detector was connected to a $10 \mathrm{kHz}$ lock-in amplifier (Brookdeal FL355) followed by a DC-recorder. The DC Stark voltage was varied such that a signal (differentiated absorption curve of the desired Stark component) appeared on the recorder. The DC-voltage corresponding to the symmetry point of the discriminator curve was measured with a compensator bridge (Bleeker-2165) calibrated against a precision volt-meter (Hewlett Packard $3420 \mathrm{~b}$, maximum $<2 \times 10^{-5}$ ).

\section{RESULTS}

The experimental method described was applied to the rotational transition $J=1 \rightarrow 2$ of OCS and particulariy the shift of the component $(J, M)=(1,0)-(2,1)$ was measured *. The

* The selection rule for transitions in the cavity is $\Delta M=$ $= \pm 1$, as the DC-electric field and the exciting microwave field are perpendicular to each other.
Shift with respect to the unsplit line $v_{0}$ was varied from $1.5 \mathrm{MHz}$ to $5 \mathrm{MHz}$. The reproducibility of Stark voltage measurements at constant frequency shift was better than $1: 10^{3}$. These measurements were repeated with five different resonant modes (different heights) of the cavity. The Stark effect of linear molecules can be described $\mathrm{b}_{\mathrm{y}}$ (see Appendix):

$$
\nu(J, M, E)=\nu_{0}+k(J, M) E^{2} \mu^{2}
$$

Thus $\mu$ and $\nu_{0}$ could be found with a least-square fit of the straight line relating $\nu$ and $E^{2}$. In this way a value for $\mu$ was obtained at each resonant mode of the cavity. The results are collected in table 2 (second column). It can be seen that a systematic variation of $\mu_{\mathrm{OCs}}$ as a function of the height is not evident; this indicates that errors due to the unevenness of the cavity-bottom and deviation from parallelism between the bottom and the plunger are vanishirgly smail. $d$ second check of the reliability of this determination was performed by repeating the measurements with a similar cavity with a diameter of $120 \mathrm{~mm}$. The results with this cavity are also shown in table 2 (fourth column). Although these results for the dipole moment show a larger scatter than those obtained with the other cavity, the values of $\mu_{\text {OCS }}$ obtained with both cavities are in agreement within the limits of experimental error. Averaging these results with a weighting factor yields:

$$
\mu_{\text {OCS }}=0.7149 \pm 0.0003 \text { Debye. }
$$

\section{CONCLUSION}

The results of this investigation agree well with the electric dipole moment data obtained

Table 2

Results of dipole moment measurements from the Stark effect of the OCS absorption line $J=1-2\left(l^{*}=0\right)$ with

\begin{tabular}{|c|c|c|c|}
\hline \multicolumn{2}{|c|}{$D=150 \mathrm{~mm}$} & \multicolumn{2}{|c|}{$D=120 \mathrm{~mm}$} \\
\hline $\mathrm{TE}_{0 m n^{-m o d e}}$ & ${ }^{\mu} \operatorname{OCS}^{a)}(D)$ & $\mathrm{TE}_{0 m i n^{-m o d e}}$ & ${ }^{\mu} \mathrm{OCS}^{2)}(\mathrm{D})$ \\
\hline 071 & $0.7153(2)$ & $0 \pm 1$ & $0.7151(3)$ \\
\hline 091 & $0.7150 \quad\left(\frac{4}{4}\right)$ & 051 & $0.7153(6)$ \\
\hline $0(10) 1$ & 0.7150 (3) & 061 & $0.7143(2)$ \\
\hline 052 & 0.7151 (3) & 071 & $0.71 \pm 8(5)$ \\
\hline \multirow[t]{2}{*}{072} & 0.7148 (I) & 042 & $0.7136\left(\frac{1}{1}\right)$ \\
\hline & & 052 & $0.71 \pm 2( \pm)$ \\
\hline \multicolumn{2}{|c|}{$\mu_{\mathrm{av}}=0.7150(2)$} & \multicolumn{2}{|c|}{$\mu_{\mathrm{av}}=0.7144(5)$} \\
\hline
\end{tabular}
two cavities of different diameter $D$.

a) The figure between brackets indicates the error in the last decimal. 
in [1] with an electric resonance beam experiment. Mor eover it has been demonstrated that accurate values of dipole moments can be obtained with a Stark absorption cavity.

\section{ACKNOWLEDGEMENT}

The authors are indebted to Professor Dr. C. Th. J. Alkemade for helpful discussions and reading of the manuscript.

This work is part of the research program of the "Stichting voor Fundamenteel Onderzoek der Materie (F.O.M.)", and was made possible by financial support from the "Nederlandse Organisatie voor Zuiver Wetenschappelijk Onderzoek (Z.W. O. )".

\section{APPENDIX}

The influence of a static electric field on the rotational energy levels of linear molecules can be determined from the perturbation

$$
H^{2}=-\mu E \cos \theta-\frac{1}{2}\left(\alpha_{\|}-\alpha_{\perp}\right) E^{2} \cos 2_{\theta}-\frac{1}{2} \alpha_{\perp} E^{2} \text {. }
$$

to the rotational Hamiltonian, where $\mu=$ electric dipole moment; $E=$ magnitude of the electric field; $\theta=$ angle between molecular axis and field applied; $\alpha_{\|}$and $\alpha_{\perp}$ are the components of the polarizability tensor indicating polarizability along and perpendicular to the molecular axis, respectively.

Since the third term yields the same correction to all rotational levels, it will not affect the transition frequency.
A first order correction from the second term and a second order correction from the first term are both proportional to the square of the electric field and yield the expression:

$$
\begin{aligned}
\Delta W_{J M}=\frac{\mu^{2} E^{2}}{2 h B} \frac{J(J+1)}{J(J+1)(2 J-1)(2 J+3)}+ & + \\
& -\frac{\alpha E^{2}}{2} \frac{2 J^{2}+2 J-2 M^{2}-1}{(2 J-1)(2 J+3)}
\end{aligned}
$$

with $\alpha=\alpha_{\text {II }}-\alpha_{\perp}$.

Using this expression and the selection rule $\Delta J=+1, \Delta M=+1$, the transition frequency of the Stark components is given by

$$
\nu(J, M, E)=\frac{W_{J+1}, M+1-W_{J, M}}{h}=\nu_{\mathrm{O}}+K(J, M)_{\mu}{ }^{2} E
$$

where the constant $k(J, M)$ contains also the small term due to $\alpha$. For the molecule OCS the polarizability anisotropy $\alpha=4.6 \times 10^{-24} \mathrm{~cm}^{3}$

[1]; the relative contribution of $\alpha$ to $k(J, M)$ is $-3.5 \times 10^{-4}$.

\section{REFERENCES}

[1] J.S. Muenter, J. Chem. Phys. 48 (1968) 4544.

[2] S. A. Marshall and J. Weber, Phys. Rev. 105 (1967) 1502.

[3] See J. E. Wollrab, Rotational spectra and molecular structure (Academic Press, New York, 1967) p. $276 \mathrm{ft}$.

[4] A. Dymanus, Physica 25 (1959) 859.

[5] H. A. Dijkerman, Ph. D. Thesis, Utrecht (1966).

[6] A. Dymanus, Rev. Sci. Instr. 30 (1959) 191. 